\title{
a-Linolenic acid induces clearance of Tau seeds via Actin-remodeling in Microglia
}

\author{
Smita Eknath Desale ${ }^{1,2}$ and Subashchandrabose Chinnathambi ${ }^{1,2^{*}}$ (D)
}

\begin{abstract}
Alzheimer's disease (AD) is known by characteristic features, extracellular burden of amyloid- $\beta$ and intracellular neuronal Tau. Microglia, the innate immune cell of the brain has the ability to clear the burden of accumulated proteins via phagocytosis. But the excessive proinflammatory cytokine production, altered cellular signaling and actin remodeling hampers the process of migration and phagocytosis by microglia. Actin remodeling is necessary to initiate the chemotactic migration of microglia towards the target and engulf it. The formation of lamellipodia, filopodia, membrane ruffling and rapid turnover of F-actin is necessary to sense the extracellular target by the cells. Omega-3 fatty acids, are known to impose anti-inflammatory phenotype of microglia by enhancing its ability for migration and phagocytosis. But the role of omega-3 fatty acids in cellular actin remodeling, which is the basis of cellular functions such as migration and phagocytosis, is not well understood. Here, we have focused on the effect of dietary supplement of a-linolenic acid (ALA) on extracellular Tau internalization and assisted actin polymerization for the process. ALA is found to induce membrane ruffling and phagocytic cup formation along with cytoskeletal rearrangement. ALA also enhances the localization of Arp2/3 complex at the leading edge and its colocalization with F-actin to induce the actin polymerization. The excessive actin polymerization might help the cell to protrude forward and perform its migration. The results suggest that dietary supplement of ALA could play a neuroprotective role and slow down the AD pathology.
\end{abstract}

Keywords: a- Linolenic acid, Lamellipodia, Filopodia, Membrane ruffling, Arp2/3 complex, Phagocytosis, Tauopathy

\section{Introduction}

Alzheimer's disease (AD) is characterized by accumulation of extracellular amyloid- $\beta$ plaques and intracellular deposition of neurofibrillary tangles (NFTs), occurred due to post-translational modification in Tau protein [1]. Tauopathy is characterized by excessive abnormal phosphorylation of Tau, which reduces its affinity towards microtubules that leads to instability in microtubule functions and loss of synaptic neurons [1-3]. Various studies have reported the ability of Tau aggregates to spread in a prion-like manner in neuronal culture through various cellular uptake mechanisms to demonstrate template-dependent aggregation of

\footnotetext{
* Correspondence: s.chinnathambi@ncl.res.in

'Neurobiology Group, Division of Biochemical Sciences, CSIR-National Chemical Laboratory, Dr. Homi Bhabha Road, Pune 411008" India

${ }^{2}$ Academy of Scientific and Innovative Research (AcSIR), Ghaziabad 201002," India
}

(c) The Author(s). 2021 Open Access This article is licensed under a Creative Commons Attribution 4.0 International License which permits use, sharing, adaptation, distribution and reproduction in any medium or format, as long as you give appropriate credit to the original author(s) and the source, provide a link to the Creative Commons licence, and indicate if changes were made. The images or other third party material in this article are included in the article's Creative Commons licence, unless indicated otherwise in a credit line to the material. If material is not included in the article's Creative Commons licence and your intended use is not permitted by statutory regulation or exceeds the permitted use, you will need to obtain permission directly from the copyright holder. To view a copy of this licence, visit http://creativecommons.org/licenses/by/4.0/.

intracellular monomeric Tau [4-6]. The extracellular species of Tau can exert toxicity in cultured hippocampal neurons via unbalanced calcium metabolism and causes cell death [7]. Microglia, the obligate phagocytes of the brain are capable of uptaking the bioactive Tau seed and neutralize it through degradation $[8,9]$. However, Microglia can contribute to Tau pathology by releasing the toxic Tau seed into the media due to its inefficiency in processing of Tau [10]. The relationship between Tau pathology and neuroinflammation by microglia is well-known, that reflects the inability of microglia to fully process the Tau seed.

Physiologically, microglia in resting state inspects the brain environment with their long extensions. The communication with other glia cells and neuron takes place via various cellular receptors, chemokine signals and soluble factors $[11,12]$. After sensing the tissue injury, pathogen invasion or plaque deposition; microglia 
becomes a motile effector cell and acquire an amoeboid form that can migrate towards a concentration gradient of chemotactic signals. For the 2-dimensional migratory motion of microglia, the cells are supported by protruding fan-shaped actin-based structures (lamellipodia) on leading edge and F-actin-rich thin extensions (filopodia) on the rear edge $[13,14]$. In this migratory state, microglia manifest the presence of podosomes, which are actin-rich structures that mediates adhesion to substratum, migration and ectracellular matrix (ECM) degradation for invasion [15]. Microglial migration followed by $\mathrm{Ca}^{2+}$ regulation is necessary for phagocytosis, and the phagocytic activity is diminished in Stromal interaction molecule (STIM) depleted microglia [16]. The initiation of signaling cascades regulating the actin cytoskeleton, which involves membrane protrusions and ruffling to increase the surface areas, are obligatory for the phagocytosis [17]. The polarized state of microglia is maintained by the cytoskeletal network, wherein actin provides directional sensing while microtubule dynamics provide mechanical strength for the forward movement of cells [18]. The co-ordinated polymerization of actin filaments provide a protrusive force to the cell for forward movement. Actin related protein 2/3 complex (Arp2/3) along with other actin-binding proteins play an essential role in nucleating the branching of actin filaments [19]. Ionized calcium-binding adaptor molecule-1 (Iba-1), a specific marker of microglia/macrophages lineage, is also reported to have a key role in the function of activated microglia $[20,21]$. In the process of membrane ruffling and phagocytic cup formation along with actin network, Iba-1 protein of microglia plays a pivotal role.

In $\mathrm{AD}$, ability of microglia to carry out phagocytosis of extracellular $A \beta$ plaques as well as aggregated Tau seeds are most affected along with the disrupted actin cytoskeleton [22]. The disrupted actin cytoskeleton due to $\mathrm{A} \beta$ via $\mathrm{A} \beta$-mediated dysregulation of phosphoinositides signaling could be consider as a cause of pathology [23]. The extracellular Tau oligomer and aggregates have the potential to remodulate the actin cytoskeleton for active phagocytosis [24]. Polyunsaturated fatty acids (PUFAs), majorly omega- 3 fatty acids have a beneficial role in the brain, which improve cognitive function and memory loss. PUFAs exist in the form of phospholipid content in the cell membrane of the brain cells, which efficiently influences the membrane receptor expression, signaling cascade and effector functions of the cell [25-27]. Omega-3 fatty acids, especially DHA and EPA exerts anti-inflammatory phenotype in the brain immune cells by modulating the lipid composition of cell membrane and cell surface receptor expression [28-30]. The cellular functions such as phagocytosis, migration are carried out with the assistance of actin remodeling [28, 31]. For the process of phagocytosis to engulf extracellular pathogens, membrane extension-induced by actin remodeling and phosphoinositides induced-signaling is imperative [32, 33]. Omega-3 PUFAs are known to significantly alter cytoskeleton-associated gene expression including Ras-related C3 botulinum toxin substrate 1 (Rac1), Cell division control protein 42 homolog (CDC42), Wiskott-Aldrich Syndrome protein (WASP) and Arp2/3 complex [28].

In this study, we aim to understand the effect of $\alpha$ Linolenic acid, a precursor of DHA and EPA, on actin remodeling related to phagocytosis and migration. In Tauopathies, extracellular Tau seeds tend to activate microglia to induce inflammatory activation. However, the study involves understanding the effect of ALA on phagocytosis and related actin-remodeling in microglia in the presence of extracellular Tau seeds. We have further studied the role of Iba- 1 and Arp2/3 complex in membrane ruffling and actin polymerization. We observed the efficiency of ALA to induce actin remodeling necessary for phagocytosis.

\section{Results}

Internalization of extracellular Tau-induced by ALA

Microglia are professional phagocytes of the brain, which assist in clearing apoptotic cells, axonal and myelin debris, central infection of bacteria and viruses, misfolded aggregated protein-like $A \beta$ and participate in synaptic pruning [34]. Along with $A \beta$ plaques, microglia also recognizes extracellular Tau oligomers and aggregates and undergo activation to engulf the Tau seeds. However, the increased production of IFN- $\gamma$ and TNF- $\alpha$ by microglia hampers its phagocytic ability and are toxic to neuronal/microglial cells [22]. PUFAs treatment reduces the production of inflammatory cytokines while exerts antiinflammatory cytokines production as well as improves phagocytosis [29]. We prepared human Tau40 (hTau40) aggregates in vitro in the presence of heparin as polyanionic agent, which can induce Tau aggregation [35]. The hTau40 aggregates were further characterized for their higher molecular weight aggregates by SDS-PAGE (Fig. 1a). The propensity of Tau aggregation was studied with ThS fluorophore that binds to Tau aggregates and gives the extent of aggregation (Fig. 1b). The fully formed mature Tau fibrils were assessed with transmission electron microscopy (TEM) (Fig. 1c). ALA was prepared by dissolving in $100 \%$ ethanol, followed by heating at $50^{\circ} \mathrm{C}$ for $2 \mathrm{~h}$ and are characterized by TEM analysis (Fig. 1d). The scheme represents the experimental proceeding of the study as Tau internalization and the changes in actin cytoskeleton associated with the process (Fig. 1e). To study the ability of ALA to cause antiinflammatory effects in microglia, we observed the phagocytosis of extracellular Tau. The N9 cells were treated with $1 \mu \mathrm{M}$ Tau monomer, $1 \mu \mathrm{M}$ Tau aggregates 
a

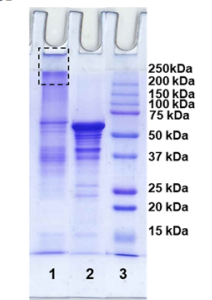

b

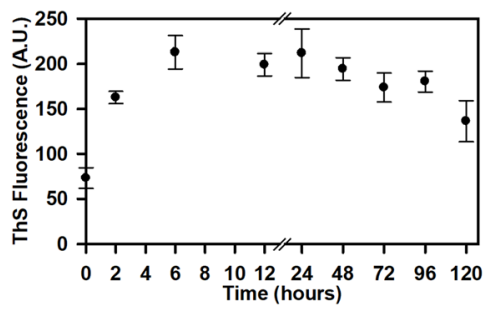

c

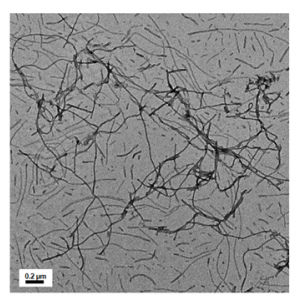

d
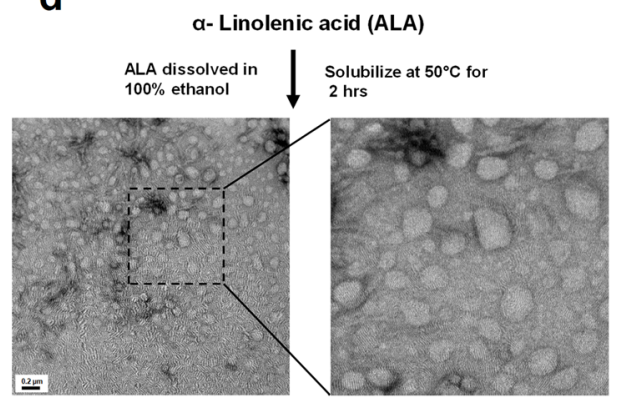

Transmission electron microscopy

e<smiles>[SiH3]</smiles>

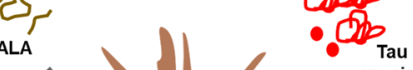

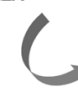

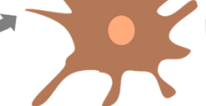

Microglia

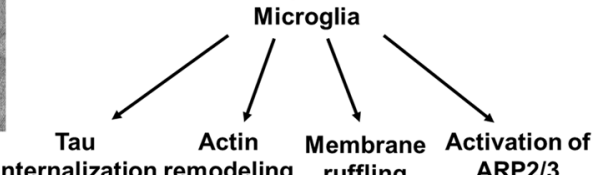

internalization remodeling ruffling ARP2/3

complex

Fig. 1 Characterization of hTau40 aggregates and experimental outline. a Human Tau40 aggregates were prepared for cellular studies. Tau aggregation was carried out in presence of heparin for $120 \mathrm{~h}$. Higher-order aggregates have been characterized by SDS-PAGE analysis. The protein band for higher-order aggregates were visible above $250 \mathrm{kDa}$ (lane 1), the protein band corresponding to monomer is indicated (lane 2). b Tau aggregates were studied by ThS fluorescence kinetics for $120 \mathrm{~h}$. c Tau aggregates were visualized via transmission electron microscopy (TEM) to understand Tau fibrils formation, scale bar is $0.2 \mu \mathrm{m}$. d ALA was prepared as a starting material in the presence of $100 \%$ ethanol and solubilized at $50^{\circ} \mathrm{C}$ for $2 \mathrm{~h}$. The transmission electron microscope image indicates the morphology of ALA, scale bar is $200 \mathrm{~nm}$. e Schematic representation of experimental proceeding depicts the role of ALA in phagocytosis of extracellular Tau and necessary actin remodeling for the process

along with $40 \mu \mathrm{M}$ ALA as a test, and respective control of Tau monomer, aggregates, ALA and cell control (untreated) were kept for comparion. Internalization of extracellular Tau in microglia was studied with fluorescence microscopy. Tau (red), Iba-1 (green) were detected in microglia cells after $24 \mathrm{~h}$ exposure of Tau and ALA to N9 microglia culture (Fig. 2a) The intracellular localization of internalized Tau in microglia are indicated in fluorescence images (Fig. 2a). The single cell and enlarged panel indicate the zoomed area of microglia with the internalized Tau, marked with the white arrow marks (Fig. 2a). The phagocytic ability of microglia for Tau monomer as well as aggregates has been observed to increase upon exposure of ALA. The mean intensity of internalized Tau has been increased to $44 \%$ for Tau monomer upon ALA exposure, whereas, the increase of $49 \%$ has been observed for Tau aggregates (Fig. 2b). The statistical analysis suggests the significance difference between Tau and ALA treated groups as compared with control (untreated) and ALA groups. The internalization ability of microglia was found to be enhanced with ALA exposure.

\section{Effect of ALA on microglial polarization and activation}

The actin cytoskeleton is involved in membraneassociated remodeling, downstream signal transduction and provides a mechanical framework for phagosomes upon internalization. Actin cytoskeleton furnishes the platform for receptors and other signaling cascades. Coordinated cycles of actin polymerization and depolymerization occurs at phagosomes for its internalization and maturation. Membrane-ruffling and phagocytic cup formation is being assisted by actin cytoskeleton along with the Iba-1 protein in microglia. Iba-1 enhances membrane-ruffling and cross-linking of actin filaments, which assist to up-regulate the internalization events [20]. After interaction of extracellular target with phagocytic receptor, signal transduction associated with actin cytoskeleton is initiated in the cell. The necessary remodeling with the membrane-associated actin and introduction of more membrane protrusion enhance the process of phagocytosis [36]. The N9 microglial cells were treated with different groups that includes $1 \mu \mathrm{M}$ hTau 40 monomer, aggregates along with their respective treatment with $40 \mu \mathrm{M}$ ALA and cell control (untreated), ALA control (only ALA treatment) was kept for the comparison. We 

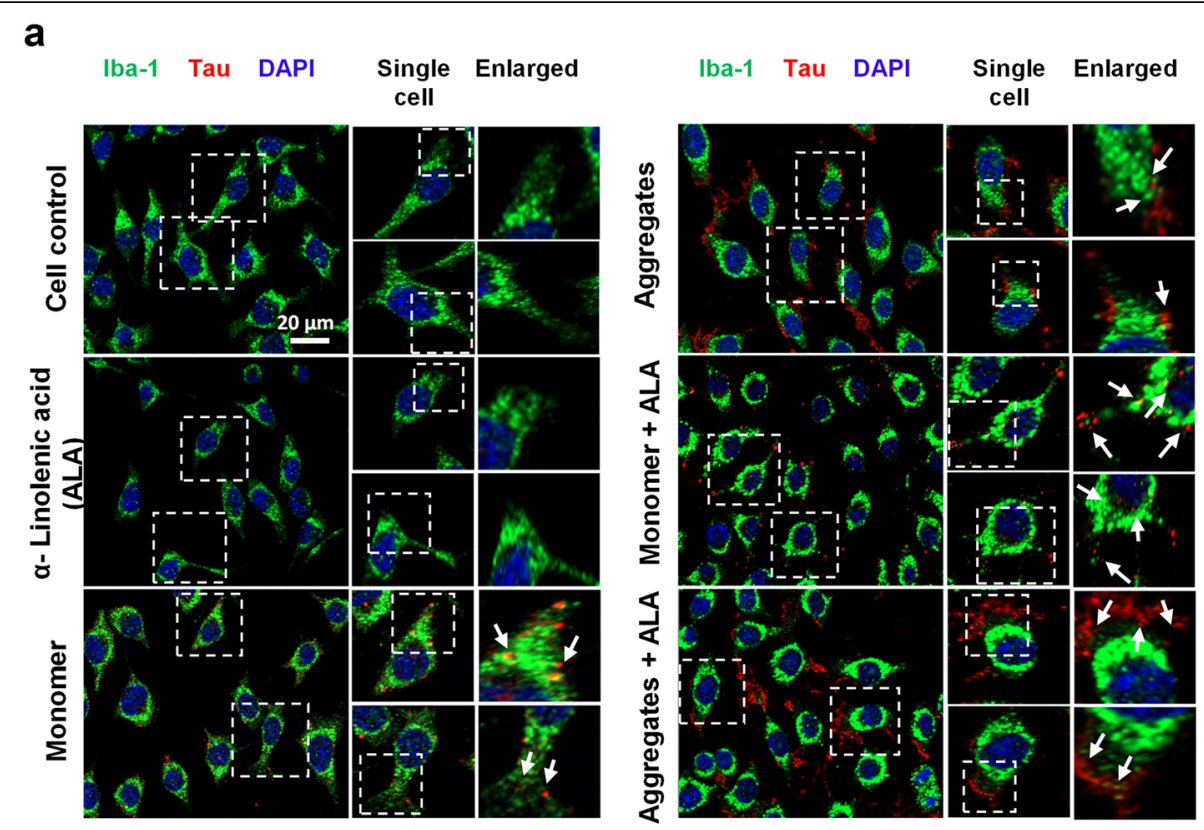

b

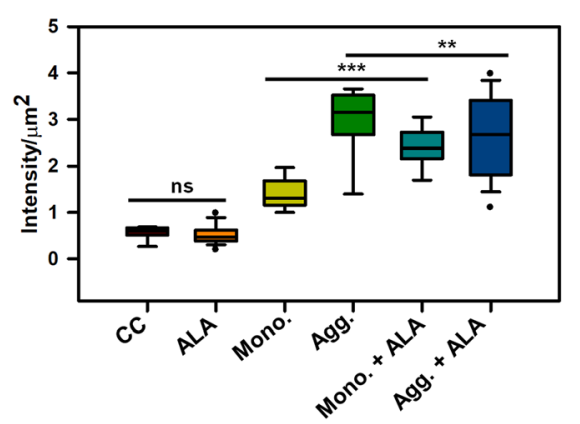

Fig. 2 Internalization of extracellular Tau in Microglia. a Study of internalization of extracellular Tau monomer and aggregates in the presence of ALA. The treatment was given for $24 \mathrm{~h}$ and studied with fluorescence microscopy to understand the internalization of Tau (red) in Iba-1 (green) positive cells. Figure showing fluorescence microscope image, single cell and enlarged panel indicates that the internalized Tau denoted with white arrow marks. The enlarged region is highlighted with white dotted square that indicates internalized area in the cells. Axio observer 7.0 Apotome 2.0 Zeiss microscope was used to the study of internalization extracellular Tau. b Quantification of internalized Tau was carried out with a total intensity of Tau (red) per $\mu \mathrm{m}^{2}$ area inside the cell was analyzed by ZEN 2.3 software. The significance was analyzed with Tukey's Kramer, significant when mean difference between treatment groups $\left(X-X^{\prime}\right)>T$ (Tukey's criteria). The significance of conditions of Tau monomer and aggregtes with ALA is $p<0.005$. annotations used in graph are as follows- CC (Cell control), ALA (a-linolenic acid), Mono. (monomer), Agg. (aggregates), Mono. + ALA (monomer plus ALA), Agg. + ALA (aggregates plus ALA)

studied the role of actin (red) and Iba-1 (green) in actinremodeling and polarization of microglia on ALA exposure by immunofluorescence staining. In the activated migratory microglia, the polymerization of actin at leading edge is signified by the presence of fan-shaped lamellipodia and thin filamentous filopodia-like structure, whereas, long extension is observed at the rear edge (Fig. 3a). For the migratory cells such as immune cells, the front-edge protrusion of lamellipodia formed by the forces of actin polymerization indicates the migratory state of microglia since they sense the extracellular Tau species. Whereas; the finger-like protrusion called as filopodia would cause the slow migration in absence of lamellipodia
[37]. The extracellular Tau can act as an activation factor for microglia. The ionized calcium-binding adaptor protein-1 (Iba-1), functions in actin-crosslinking in membrane ruffling, which plays a role during ramified to amoeboid change in microglia. This possibly relate the activation of microglia with increased expression of Iba-1 [38]. The immunofluorescence staining indicates enhanced colocalization between Iba- 1 and actin at the leading edge of activated microglia cells in ALA treated groups (Fig. 3a). The expression of Iba- 1 has found to be increased upon exposure of Tau as compared to cell control (untreated) that can notify the activation state of microglia (Fig. 3b, c, d). These results indicate the 


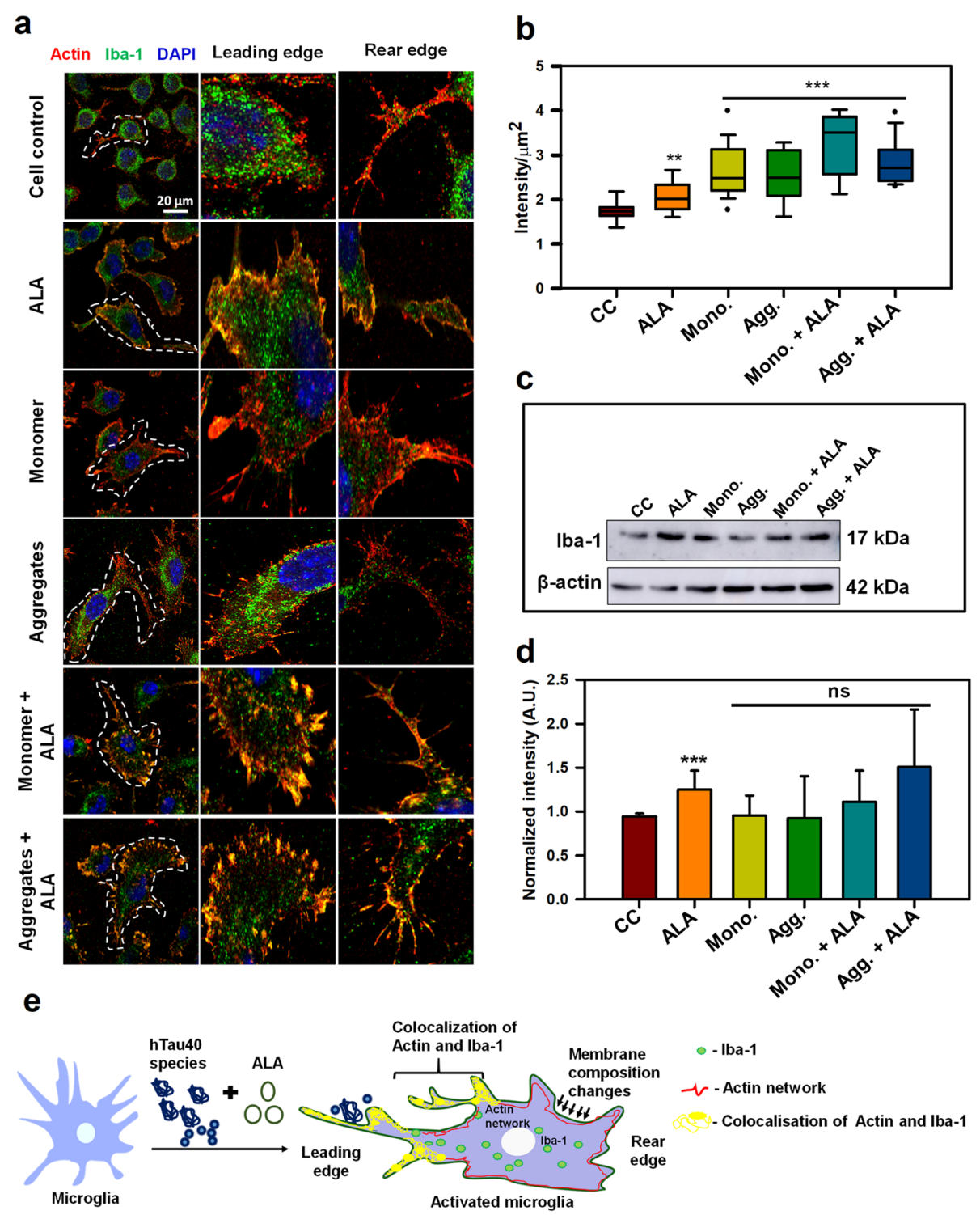

Fig. 3 Modulation of actin network in activated microglia by ALA. Role of actin and Iba-1 in microglial migration and activation a N9 microglial cells were treated with Tau monomer, aggregates along with ALA for $24 \mathrm{~h}$. After fixation cells were stained with $\beta$-actin (red) and anti-lba-1 antibody (green). Colocalization of actin and Iba-1 was studied by Super-resolution confocal microscopy. The single cells are marked with white dotted lines in the merged image, scale bar is $20 \mu \mathrm{m}$. The migratory microglia are shown with the front; leading edge and rear edge and the colocalization of actin and Iba-1 in the edges assisting the microglia for membrane ruffling and phagocytosis. $\mathbf{b}$ The quantification of Iba-1 in cell indicating its activation status and its levels on increased phagocytosis. The increased levels of total intensity per $\mu m^{2}$ area of microglia was calculated with ZEN 2.3 software, significance is $(p<0.001)$. c The protein expression of Iba-1 was measured by western blot analysis in all the treated groups after $24 \mathrm{~h}$ of treatment and stained with anti-lba-1 and $\beta$-actin antibody for loading control. $\mathbf{d}$ Quantification of the intensity of protein bands from western blot normalized with the $\beta$-actin loading control. The conditions of Tau monomer and aggregtes with ALA are nonsignificant, $(p>0.05)$. e Schematic overview of actin remodeling at leading edge of microglia and involvement of Iba- 1 in the process of phagocytosis after ALA treatment to cells. The significance was analyzed with Tukey's Kramer, significant when mean difference between treatment groups $\left(X-X^{\prime}\right)>T$ (Tukey's criteria). The annotations used in graph are as follows- CC (Cell control), ALA (a-linolenic acid), Mono. (monomer), Agg. (aggregates), Mono. + ALA (monomer plus ALA), Agg. + ALA (aggregates plus ALA).

involvement of actin and Iba-1 in phagocytic cup formation and membrane ruffling (Fig. 3a, 4a) [20]. Intracellular intensity of Iba-1 was quantified via ZEN 2.3 software and it was found to increase significantly in both monomer and aggregates in presence of ALA. The statistical analysis suggest the significant difference among groups treated with Tau and ALA as compared with control (untreated) and ALA groups $(p<0.001)$ and the data was plotted as mean with the standard error between the individual conditions (Fig. 3b). Iba-1 levels were also quantified by 

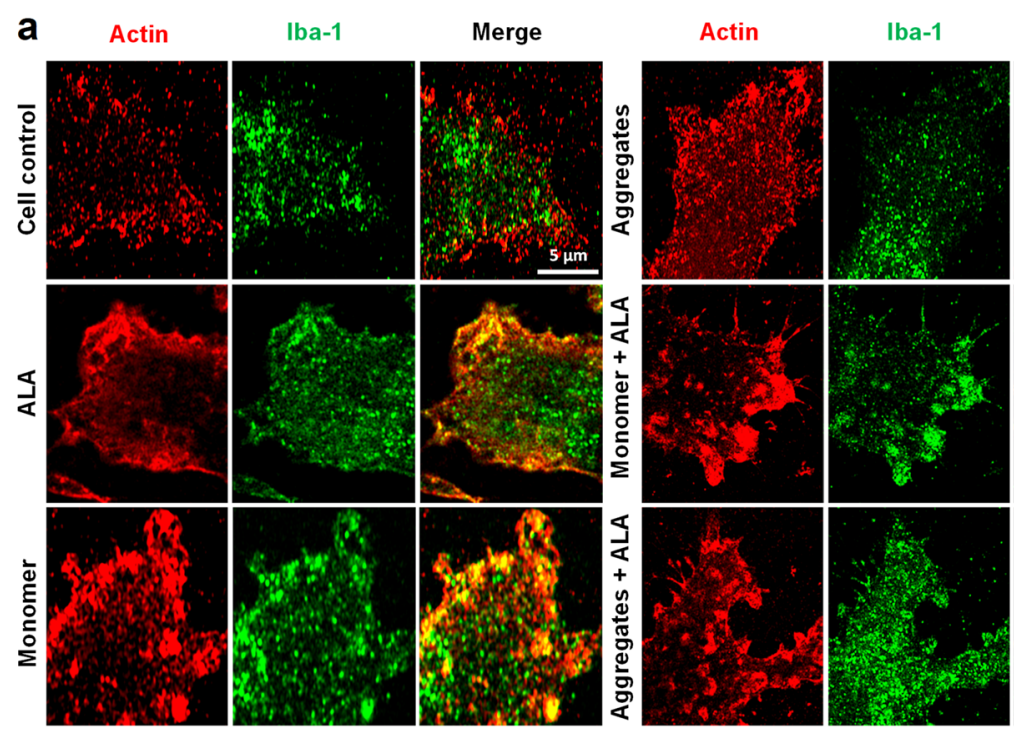

Merge

b

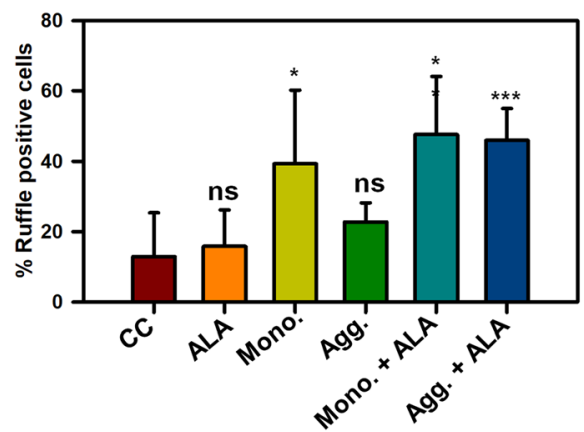

Fig. 4 Formation of membrane ruffles induced by ALA. Role of actin and Iba-1 in the formation of membrane ruffling was studied upon exposure of ALA. The effect of ALA in membrane ruffling was observed with the super-resolution confocal microscopy. a N9 cells were treated with Tau monomer, aggregates and ALA for $24 \mathrm{~h}$ followed by staining with actin and Iba-1 to study the membrane ruffling. The zoom images are indicated with the colocalization of actin and Iba-1 showing membrane ruffling in images. The sacel bar is $5 \mu \mathrm{m}$. $\mathbf{b}$ The quantification of ruffle positive cells by measuring actin foci per cell were carried out for the percentage calculation. The conditions of Tau monomer and aggregtes with ALA are significant, $(p<0.005)$. The significance was analyzed with Tukey's Kramer, significant when mean difference between treatment groups $\left(X-X^{\prime}\right)>T$ (Tukey's criteria). The annotations used in graph are as follows- CC (Cell control), ALA (a-linolenic acid), Mono. (monomer), Agg. (aggregates), Mono. + ALA (monomer plus ALA), Agg. + ALA (aggregates plus ALA)

western blot, Iba-1 levels have been increased with respect to cell control (untreatred), which is presented as the quantification with loading control ( $\beta$-Actin) (Fig. $3 \mathrm{c}$ and d). The levels of Iba-1 although found to increase but data is found to be insignificant after post-hoc analysis. In this study, we observed that, exposure of ALA and Tau species to microglia triggers the microglial activation and enhance actin remodeling, which may induce processes such as migration and phagocytosis. Actin and Iba-1 play a major role in microglial migration and activation, which assist the phagocytosis (Fig. 3e).

\section{Enhancement of membrane ruffling in presence of ALA} After the detection of the target, immune cells increase the membrane protrusion and membrane ruffling to increase the area in contact with the target to initiate the internalization process. Importance of actin polymerization and involvement of Iba-1 in membrane ruffling has been studied with Super-Resolution Confocal microscopy. The presence of ALA was found to enhance the membrane ruffling in both monomer and aggregates treated cells, which might enhance the chances of internalization in microglia, scale bar is $5 \mu \mathrm{m}$ (Fig. 4a). The overall quantification of membrane ruffle positive cells were carried out with the number of actin foci per cell according to previous studies [39]. The quantification indicates the increased occurrence of membrane ruffle positive cells upon ALA exposure in both Tau monomer and aggregates treated cells. The statistical analysis suggest the significance difference 
amongst ALA and Tau treatment groups as compared to control (untreated) and ALA groups $(p<0.005)$ and the data was plotted as a mean with standard error between the conditions (Fig. 4b). The overall view of the zoomed images with separate panel is shown in supplementary figure, scale bar is $20 \mu \mathrm{m}$ (Fig. S1). Respective cell have been marked with dotted line from where the enlarge areas have been observed.

\section{ALA enhance actin structure for migration and phagocytosis}

Immune cells are characterized by excessive motility to survey environment to find and destroy target pathogens. Actin polymerization provides necessary protrusion for the cells to move forward, which can be observed with the lamellipodia and filopodia to sense distant targets [19]. The actin-remodeling is important for phagocytosis, motility as well as matrix degradation [18]. We observed the role of actin-rich structures lamellipodia and filopodia in migration and phagocytosis. N9 cells were stained with F-actin (Phalloidin) to understand the fine actin structures (Fig. 5a). The sheet-like membrane protrusions at the leading edge and fingerlike protrusions were visualized with phalloidin staining after $24 \mathrm{~h}$ exposure of Tau monomer, aggregates along with ALA. The lamellipodia-like front -edge protrusions have been increased upon Tau treatment along with ALA, which indicates ALA initiates the motility and phagocytosis of extracellular Tau $(p<0.001)$ (Fig. 5a, b). The data is significant as compared to control (untreated), ALA groups. The quantification of percentage lamellipodia positive cells have been performed on 10 different microscopic fields. The cells showing fanshaped protrusion at the leading edge were considered as a lamellipodia positive cell $[24,40]$. The actin spike, which are known as filopodia-like structures and the number of filopodia per cell have been increased in ALA exposed groups indicating activation and initiation of motility of microglia in the presence of ALA $(p<0.001)$ (Fig. 5a, c, d). The filopodia positive cells and number of filopodia per cells have been calculated as previous studies, any point protrude from the cell and more than $1 \mu \mathrm{m}$ is considered as filopodia [24, 41]. The percentage filopodia positive cells have increased over ALA and Tau exposure of both monomer and aggregates, as compared to control groups (Fig. 5c). Statistical analysis suggests that, there is clear significant difference between Tau and ALA treated groups as compared with control (untreated), ALA groups. The number of filopodia per cell has increased significantly with ALA exposure in monomer and aggregates treatment, and the numbers of filopodia per cell are maximum in monomer with ALA treatment $(p<0.001)$ (Fig. $5 \mathrm{~d})$. The significance analysis indicated the significant difference between ALA and
Tau treated groups with respect to control (untreated), ALA treated groups. Actin plays a major role in phagocytosis and migration, which can be observed by enhanced actin structures in the form of lamellipodia and filopodia upon ALA exposure. Therefore, the study suggested an independent beneficial effect of ALA as a omega-3 fatty acid on actin-remodeling.

\section{ALA enhances actin branching network}

The nucleation of actin filaments is required to start the assembly process of any actin-based structures. The protrusive edge of cell is especially enriched with orthogonally cross-linked actin filaments, which are induced due to the presence of assembly factors such as, Arp $2 / 3$ complex [42]. The protrusive force needed for the cell to move forward is provided by actin polymerization, where Arp2/3 complex plays a larger role to initiate the polymerization process. The increased density of Arp $2 / 3$ complex at the membrane of extending lamellipodia suggest the involvement of Arp2/3 complex in the formation of growing filaments [43]. We have studied the occurrence of Arp2/3 complex in microglia after treatment of Tau monomer, aggregates and ALA. The representation of images indicate Arp2/3 (red), phalloidin (green) and DAPI-nuclear staiming (blue) indicates the presence of Arp2/3 at the leading edge of cells, inducing more branching of actin filaments for the protrusive force (Fig. 6a). The single cell and enlarged panel indicates that colocalization of Arp $2 / 3$ complex with F-actin, and the degree of colocalization have found to increase with ALA exposure to cells. The degree of colocalization between F-actin and Arp2 have found to increased in test groups treated with Tau species and ALA, as compared to control groups (Fig. 6a). Figure S2 indicates the orthogonal section showing intracellular colocalization of F-actin and Arp2/3 in XY plane (Fig. S2). Intensity of Arp2/3 complex protein was calculated from immunofluorescence images to check the protein levels. The fluorescence intensity (A.U.) of Arp2/3 indicates that the levels have increased in the presence of monomer plus ALA, however ALA control also showed increase in the intensity of Arp2/3 $(p<0.001)$ (Fig. 6b). The statistical analysis suggests the significant difference between control (untreated) and Tau and ALA treated groups. To further understand the colocalization index between Arp2/3 and F-actin, the Pearson's coefficient for colocalization was calculated using ROI, and the analysis indicated an increased degree of colocalization in test groups treated with Tau monomer, aggregates along with ALA $(p<0.001)$ (Fig. 6c). The statistical analysis suggests that the Tau and ALA treated groups are significant with respect to control groups (untreated and ALA). The protein expression of Arp2/3 was also estimated by western blot to understand the changes in 
a
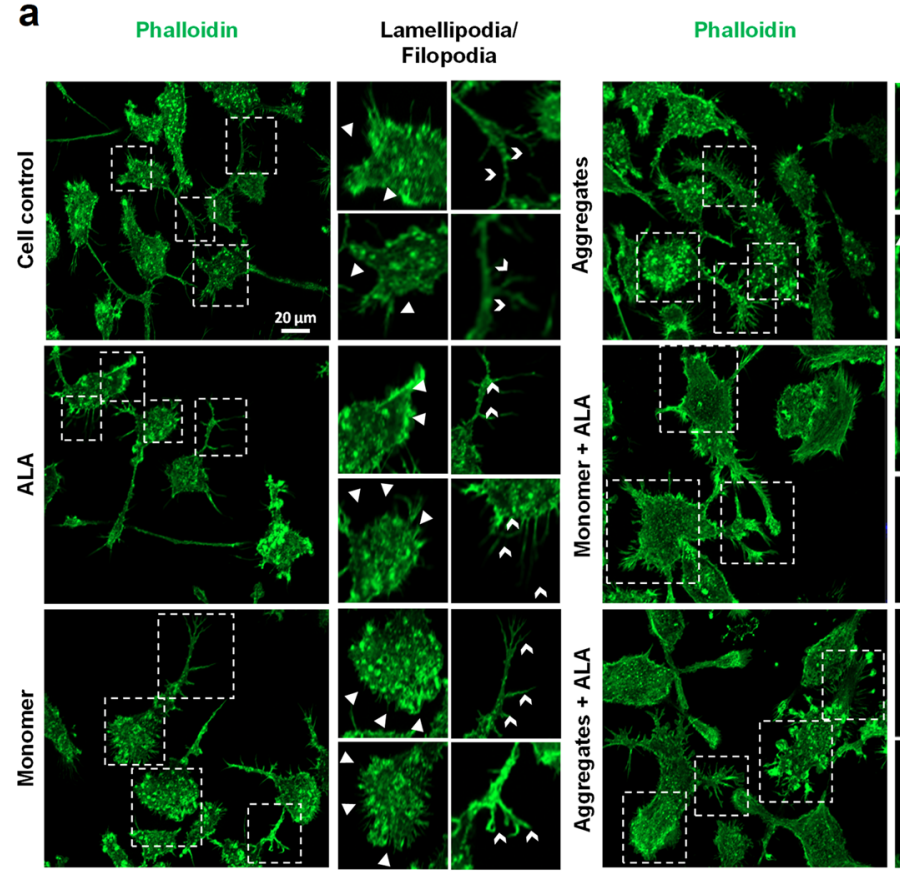

Lamellipodia

Filopodia

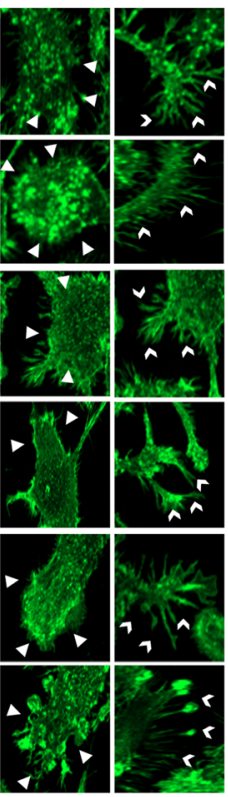

d
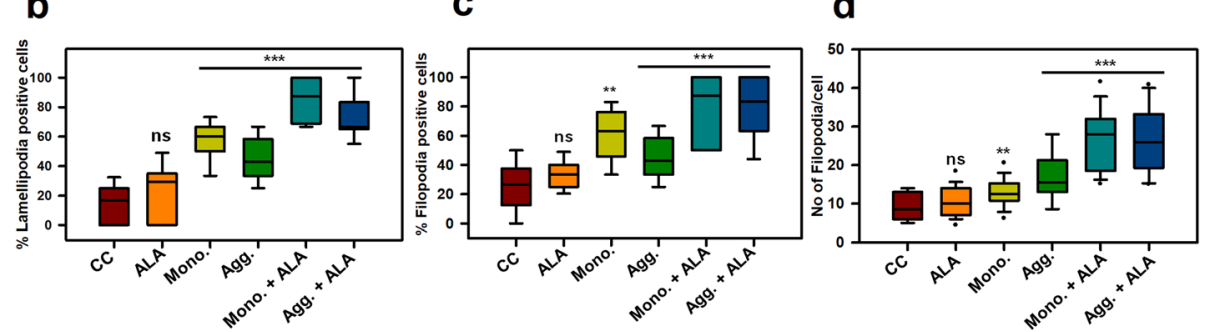

Fig. 5 Enhancement of actin-rich structures for migration in microglia. Actin plays a significant role in the migration of microglia by providing mechanical strength as well as direction support. a Actin based structures lamellipodia, filopodia were observed after $24 \mathrm{~h}$ exposure Tau monomer, aggregates along with ALA to microglia cells by fluorescence microscopy. The enlarge panel indicates the presence of lamellipodia (white triangles), filopodia (white arrow heads) from the fluorescence image. The enlarged regions are denoted with the white dotted square in the image, scale bar is $20 \mu \mathrm{m}$. b The graph represents quantification of percentage of lamellipodia positive cells per 10 fields of different treatment groups, $(p<0.001)$ as compared to cell control (untreated) and ALA. $\mathbf{c}$ The graphical representation of percentage of filopodia positive cells per 10 fields of different treatment groups Tau monomer, aggregates along with ALA showed high significance of $(p<0.001) \mathbf{d}$ Quantification of number of filopodia extensions present per cell per 10 fields of different groups, significance is $(p<0.001)$. The significance was analyzed with Tukey's Kramer, significant when mean difference between treatment groups $\left(X-X^{\prime}\right)>T$ (Tukey's criteria). The annotations used in graph are as follows- CC (Cell control), ALA (a-linolenic acid), Mono. (monomer), Agg. (aggregates), Mono. + ALA (monomer plus ALA), Agg. + ALA (aggregates plus ALA)

expression pattern or the protein density per area. The western blot analysis indicated the increased levels of Arp2/3 in test groups treated with Tau monomer, aggregates along with ALA (Fig. 6d). The quantication of intensity of protein bands normalized to loading control ( $\beta$-tubulin) was found to increased over Tau and ALA exposure (Fig. 6e). The statistical analysis suggests that the Tau and ALA treated groups are statistically significant with respect to control (untreated) group. The overall results suggest that ALA can involve in the activation of Arp2/3 to induce actin polymerization.

\section{Discussion}

Extracellular deposits of $A \beta$ and intracellular hyperphosphorylated neurofibrillary tangles of Tau depict the hallmark of AD. Accumulation of deposits of $\mathrm{A} \beta$ and aggregated Tau seeds in the brain environment prompt cognitive decline and neuronal loss [44]. Microglia as brain macrophages screen the brain environment by constantly elongated processes in the resting stage. After encountering the site of injury or protein deposits, they extend the processes and migrate towards the recognized site, ramified in shape and exert an immune 
a

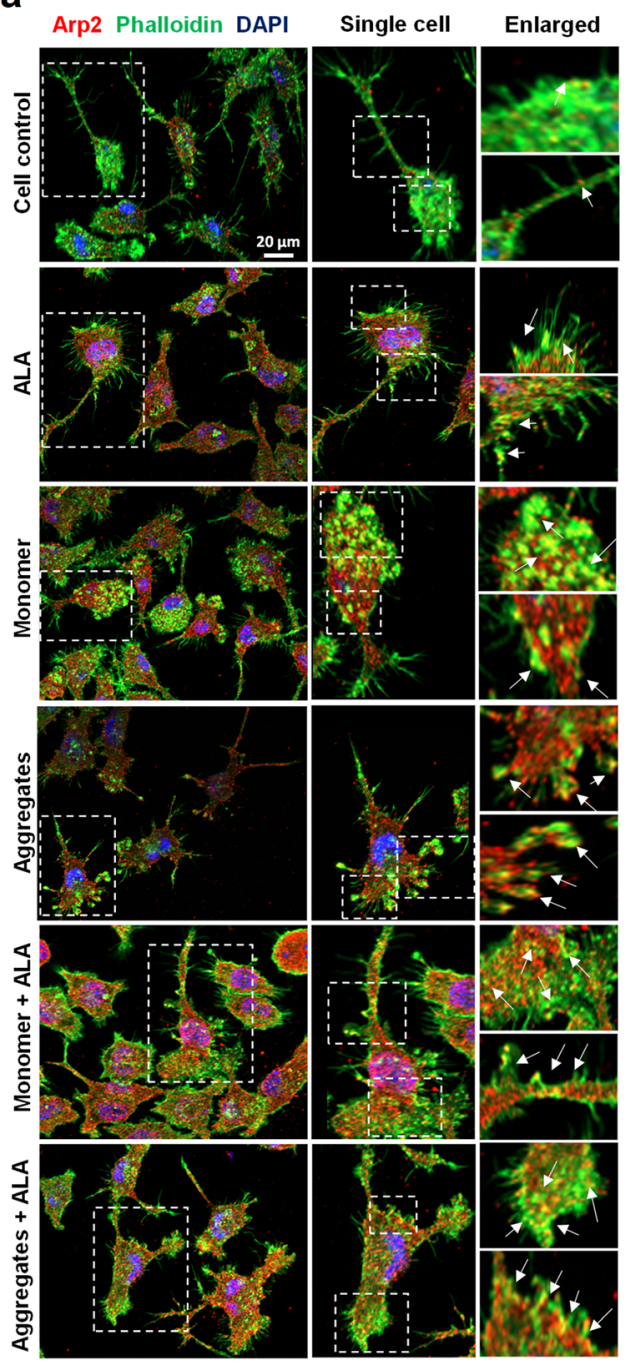

\section{b}

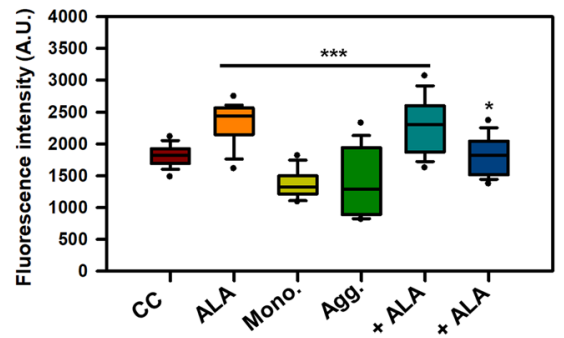

C
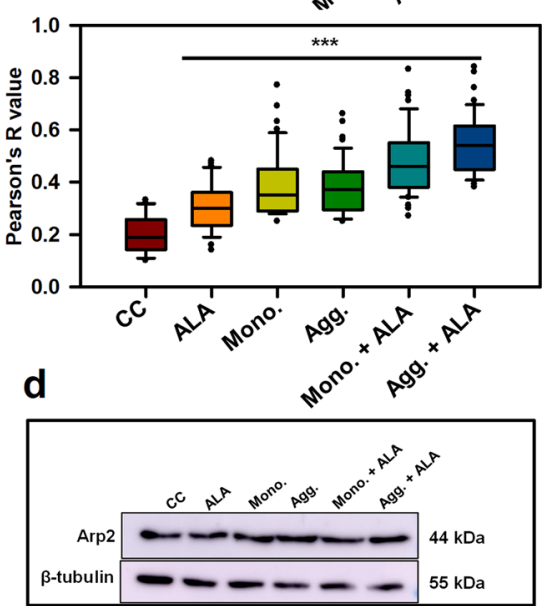

e

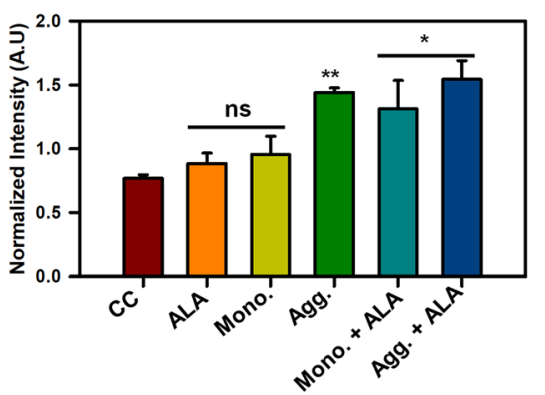

Fig. 6 Enhancement of Arp2/3-mediated actin polymerization in the presence of ALA. Arp2/3 complex plays an important role in nucleating the actin polymerization and provide pushing force for the cell to move forward. After exposure of Tau monomer, aggregates and ALA the abundance of Arp2/3 in microglia was studied by fluorescence microscopy. a Immunofluorescence images indicate the microglia cells stained with Arp2/3 (red), phalloidin (green), DAPI, scale bar is $20 \mu \mathrm{m}$. The enlarged area indicates the colocalization of F-actin and Arp2/3 complex. The enlarged area has been taken from single cell panel and marked with white dotted squares. The white arrow marks indicates concentrated colocalized area inside the cell. $\mathbf{b}$ The intracellular intensity of Arp2/3 in the cell was quantified from immunofluorescence images with ZEN 2.3 software and plotted as a fluorescent intensity units $(p<0.05)$ for ALA treated groups as compared to cell control. c The Pearson's R value for colocalization was calculated with ImageJ software. The conditions of Tau monomer and aggregtes with ALA are significant, $(p<0.001)$. $\mathbf{d}$ The quantification of protein levels of Arp2/3 was observed from western blot analysis after $24 \mathrm{~h}$ treatment of Tau monomer, aggregates and ALA. e The graph indicates quantification of western blot bands normalized with the loading control $\beta$-tubulin. The conditions of Tau monomer and aggregtes with ALA are significant, $(p<0.05)$. The significance was analyzed with Tukey's Kramer, significant when mean difference between treatment groups $\left(X-X^{\prime}\right)>T$ (Tukey's criteria). The annotations used in graph are as follows- CC (Cell control), ALA (a-linolenic acid), Mono. (monomer), Agg. (aggregates), Mono. + ALA (monomer plus ALA), Agg. + ALA (aggregates plus ALA)

response. In $\mathrm{AD}$, microglia surrounds the $\mathrm{A} \beta$ plaques, activates and produce inflammatory response [45]. The gathered microglia towards senile plaques induce the formation of $\mathrm{A} \beta$ deposits rather than clearance due to excessive inflammatory cytokines such as IFN- $\gamma$ and
TNF- $\alpha$ [22]. Iba-1 positive microglia also found to accumulate near NFT bearing neurons and induce inflammatory phase [46]. Tau seeds have the ability to propagate in a "prion-like" manner and efficiently alter microglial activation to fabricate inflammatory response $[5,47,48]$. 
In this condition, inducing anti-inflammatory phenotype of microglia could act as therapeutic strategy and omega-3 fatty acids speed up the process [26]. Omega-3 PUFAs on dietary intake improves cognitive impairment, apoptosis of neurons and inflammatory phase by microglia [25]. DHA and EPA exert polarization of M2 phenotype of microglia along with the excessive expression of phagocytic receptors such as CD206 and antiinflammatory cytokine IL-4, IL-10 etc. [29, 49]. The cytoskeleton changes needed for the phagocytosis by microglia is also induced via omega-3 PUFAs by various mechanism including phosphoinosites signaling [23]. Our results suggest that the necessary actin remodeling for migration and phagocytosis assists the phagocytosis of extracellular Tau species induced by ALA. Coordinated polymerization of a branched network of actin filaments is a key phenomenon to generate pushing force for the migratory cells. Extensive motility of immune cells is necessary to locate a target to destroy. The identification of targets via receptors initiates a signaling cascade involving actin polymerization to produce membrane protrusions around the target to engulf. After engulfment, the target is enclosed in endocytic vesicle and pinched out from the cell membrane. In this process, actin remodeling plays a major role to induce changes in the plasma membrane, membrane ruffling and protrusions that speed-up phagocytosis $[19,50]$. Membrane ruffling shows the involvement of Iba- 1 along with actin, whereas Iba-1 mutants hamper membrane ruffling and phagocytic cup formation [20]. The role of Iba-1 as an actin-binding protein has been studied. The activated microglia after $24 \mathrm{~h}$ of treatment of ALA and Tau showed high colocalization between actin and Iba-1 at the site of protrusive membrane and enhanced membrane ruffling, lamellipodia and filopodia. ALA was found to increase membrane ruffles to a great extent, which would be effective for increased phagocytosis. Previous studies have been reported for the physical binding of Iba-1 to F-actin, and their involvement in membrane ruffling supports the increased colocalization of Iba-1 and actin in ALA treated cells. These results suggest that the importance of ALA to enhance microglial activation in the presence of Tau species. The activated microglia in the presence of Tau species and ALA might suggest the involvement of ALA as an inducer of phagocytosis to clear the extracellular debris. After CNS injury, microglia has tendency to migrate towards the site of injury and further the migration is supported by rearrangement of actin cytoskeleton [51]. The actin structures and dynamics support migration, whereas lamellipodia structure produces forward protrusive forces and thin protrusion of filopodia inspect distant targets. The ALA treated cells showed greater polarized morphology with presence of dense lammellum at leading edges and uropods at rear edges. The lammellum and filopodia extensions were high in ALA treated cells, which suggests a high directional migration and supports protrusive and contractile migration as microglia sense the extracellular Tau. In previous studies, with the alternative activation of microglia by IL- 4 treatment, lammellum with more membrane ruffles have been observed [18]. Similar morphology was observed with ALA treated cells. The actin filament polymerization required for lamellipodia formation is nucleated by Arp $2 / 3 \mathrm{com}$ plex. Arp $2 / 3$ introduces branching of mother actin filaments to produce a dense branching network of the actin. The density of Arp $2 / 3$ in migratory cells is higher at leading edge to produce dense actin network and fade out in other cell body $[19,50]$. The increased colocalization between F-actin and Arp $2 / 3$ at the leading edge, might suggest the importance of ALA as an inducer of actin polymerization necessary for migration to reach out to extracellular debris. ALA found to enhance Arp2/ 3 at the leading edge, since it enhances lamellipodia formation. This depicts that ALA has tendency to remodulate actin network (Fig. 7).

\section{Conclusions}

The necessary actin-remodeling for migration and phagocytosis to grasp the target and neutralizing it is one of the therapeutic approach to increase the phagocytic efficiency of microglia. ALA effectively improves the actin remodeling by enhancing lamellipodia, filopodia as well as membrane ruffling. Arp2/3 complex, a nucleation factor for actin polymerization is shown to colocalize with F-actin, which help to improve F-actin turnover. Improved actin remodeling in the presence of ALA may increase the chances of microglia to target the extracellular burden of Tau. Therefore, including ALA as a dietary supplement might be a potential therapeutic approach to clear Tau seeding.

\section{Materials and methods \\ Chemicals and primary antibodies}

Luria-Bertani broth (Himedia); Ampicillin, $\mathrm{NaCl}$, Phenylmethylsulfonylfluoride (PMSF), $\mathrm{MgCl} 2, \quad$ APS, DMSO, Ethanol (Mol Bio grade), Isopropanol (Mol Bio grade) and methanol (Mol Bio grade) were purchased from MP biomedicals; IPTG and Dithiothreitol (DTT) from Calbiochem; MES, BES, SDS, $\alpha$-Linolenic acid (ALA) (L2376) from Sigma; EGTA, Protease inhibitor cocktail, Tris base, 40\% Acrylamide, TEMED from Invitrogen. For cell culture studies, the N9 microglial cell line no. is CVCL- 0452, Roswell Park Memorial Institute (RPMI), Fetal Bovine Serum (FBS), Horse serum, Phosphate buffer saline (PBS, cell biology grade), Trypsin-EDTA, Penicillin-streptomycin, 


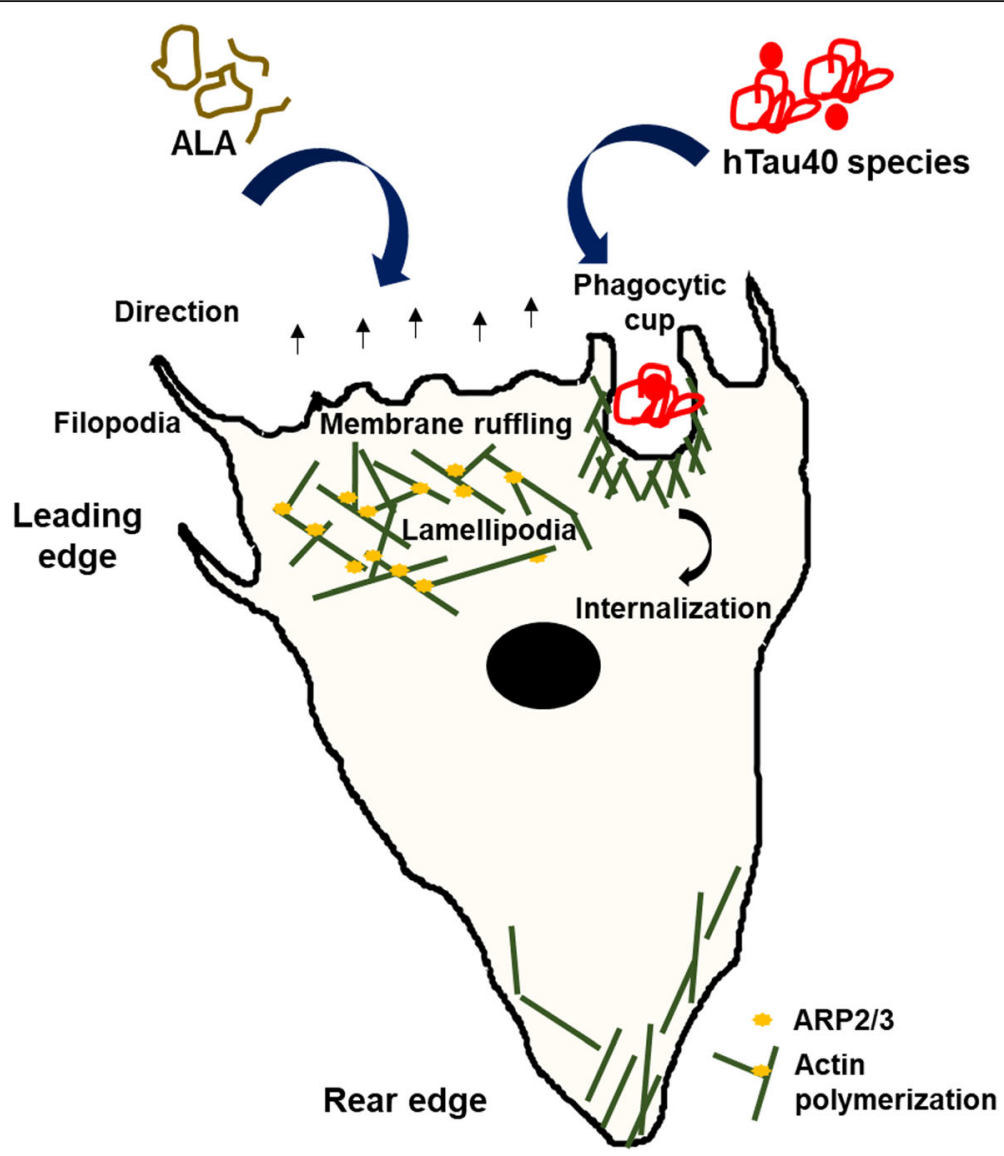

Fig. 7 a-Linolenic acid as a modulator of actin cytoskeleton for phagocytosis and migration of microglia. The picture representation suggests effects of ALA after exposure to microglial cells in enhancing extracellular Tau phagocytosis and associated actin-remodeling. ALA enhances phagocytosis of microglia with increased actin dynamics, the actin structure lamellipodia, and filopodia for the migration and promotes membrane ruffling to support the process of phagocytosis

RIPA buffer were also purchased from Invitrogen. MTT reagent and Triton X-100, Trypan-Blue were purchased from Sigma. The coverslip of $12 \mathrm{~mm}$ and $18 \mathrm{~mm}$ was purchased from Bluestar for immunofluorescence and copper-coated carbon grids for TEM analysis were purchased from Ted Pella, Inc. In immunofluorescence and western blot study we used the following antibodies: $\beta$-actin (Thermofisher cat no. MA515739), ARP2 MONO (Thermofisher cat no703394), Anti-Iba-1 (Thermo cat no-PA527436), Alexa Fluor-488 Phallodin (A12379), anti-mouse secondary antibody conjugated with Alexa Fluor-488 (Invitrogen, cat no A-11001), Goat anti-Rabbit IgG $(\mathrm{H}+\mathrm{L})$ CrossAdsorbed Secondary Antibody with Alexa Fluor 555 (A-21428), GOXMS ALEXA FLOUR 488 goat anti rabbit (Thermofisher cat no A28175) DAPI (Invitrogen), Goat Anti Mouse secondary antibody Peroxidase conjugated (Thermo fisher 32,430), Prolong Diamond antifade (Thermofisher cat no- P36961).

\section{Protein expression and purification}

Full-length wild type Tau protein $\left(\mathrm{hTau} 40^{\mathrm{wt}}\right)$ was expressed in BL21* cells with $100 \mu \mathrm{g} / \mathrm{ml}$ of ampicillin antibiotic selection and purified with two-step chromatography methods, cation-exchange chromatography and size-exclusion chromatography [52]. Induction was carried out with $0.5 \mathrm{mM}$ IPTG for $3 \mathrm{~h}$ at $37^{\circ}$. In brief, the cell lysate was subjected to $90{ }^{\circ} \mathrm{C}$ heating and supernatant was centrifugation at $40000 \mathrm{rpm}$ for $45 \mathrm{~min}$ followed by dialysis overnight at $4{ }^{\circ} \mathrm{C}$ in $20 \mathrm{mM}$ MES buffer. hTau40 purified by cation-exchange chromatography with Sepharose fast-flow column was used for chromatography. Fractions containing Tau proteins were collected after cation exchange chromatography, it was then concentrated and subjected to size-exclusion chromatography. Size-exclusion chromatography was carried out in the Superdex 75 Hi-load 16/600 column in 1X PBS supplemented with $2 \mathrm{mM}$ DTT. Fractions containing Tau were collected, pooled, concentrated, and the 
concentration of protein was determined with Bicinchoninic acid (BCA) assay.

\section{Aggregation assay}

Natively unfolded protein Tau undergo aggregation in the presence of poly-anionic agent heparin or arachidonic acid to produce $\beta$ - sheet structure $[35,53]$. Tau aggregation was induced by heparin (MW-17500 Da) in the ratio of 1:4 (heparin to Tau) along with other additives $20 \mathrm{mM}$ BES buffer, $25 \mathrm{mM} \mathrm{NaCl}, 1 \mathrm{mM}$ DTT, $0.01 \% \mathrm{NaN}_{3}, 20 \mu \mathrm{l}$ of PIC. Aggregation propensity of Tau was checked with ThS, is a homogeneous mixture of methylation product of dehydrothiotoluidine in sulfonic acid, which can bind to the $\beta$-sheet structure. Aggregation kinetics of Tau was studied with $2 \mu \mathrm{M}$ of Tau and ThS in 1:4 ratios. The excitation wavelength for $\mathrm{ThS}$ is $440 \mathrm{~nm}$ and the emission wavelength is $521 \mathrm{~nm}$. Three-independent experiments have been performed and further data analysed using Sigmaplot 10.0.

\section{Transmission electron microscopy}

Tau fibrils and ALA vesicles were studied by transmission electron microscopy (TEM) for morphological analysis. $2 \mu \mathrm{M}$ Tau sample was incubated on 400 mesh, carbon-coated copper grid for observation and stained with 2\% Uranyl acetate for the contrast. For ALA vesicles working concentration of $40 \mu \mathrm{M}$ was taken from the previous studies, for grid preparation [29, 35, 54]. The microscopic observation of Tau fibrils and ALA was carried out by TEM TECNAI T20 120KV.

\section{Cell culture condition}

N9 microglial cells were grown in RPMI media supplemented with $10 \%$ heat-inactivated serum, $1 \%$ penicillinstreptomycin antibiotic solution and glutamine and grown in T25 flask or $60 \mathrm{~mm}$ dish to maintain the culture. Cells were passaged using $0.25 \%$ trypsin-EDTA solution after washing with PBS, on attaining $90 \%$ confluency or more. For western blot experiment cells were seeded in 6 well plate, while for immunofluorescence experiment cells has been seeded in 12 or 24 well plate on to a coverslip. For $\alpha$-Linolenic acid preparation, our previously published protocol was followed [29]. Briefly, ALA was dissolved in $100 \%$ molecular biology grade ethanol and solubilized at $50^{\circ} \mathrm{C}$ in the stock concentration of $20 \mathrm{mM}$. ALA solution prepared fresh before every experiment. The working concentration of ALA $40 \mu \mathrm{M}$ was decided according to previous studies, and the final concentration of ethanol in the culture was maintained below $0.5 \%$ to avoid solvent-mediated toxicity $[29,35,54]$.

\section{Immunofluorescence analysis}

For immunofluorescence 25,000 cells of N9 microglia were seeded on $12 \mathrm{~mm}$ coverslip (Bluestar) in 24 well plate supplemented with $10 \%$ FBS and $1 \%$ penicillinstreptomycin. During treatment of hTau40 monomer/ aggregates and ALA the cells were supplemented with $0.5 \%$ serum-deprived RPMI media. The treatment of hTau 40 monomer, aggregates, ALA was given for $24 \mathrm{~h}$. Cells were then fixed with $4 \%$ paraformaldehyde solution for $20 \mathrm{~min}$ at room temperature then washed with $1 \mathrm{X}$ PBS thrice. Permeabilisation before staining was carried out using $0.2 \%$ Triton X-100 for $15 \mathrm{~min}$ followed by washing three times with $1 \mathrm{X}$ PBS and blocking with $2 \%$ serum in $1 \mathrm{X}$ PBS for $1 \mathrm{~h}$ at room temperature. Primary antibody/phalloidin solution treatment was given to cells overnight at $4{ }^{\circ} \mathrm{C}$ in $2 \%$ serum in $1 \mathrm{X}$ PBS in a moist chamber. The next day, cells were washed with $1 \mathrm{X}$ PBS thrice. Then incubated with secondary antibody in $2 \%$ serum at $37^{\circ} \mathrm{C}$ for $1 \mathrm{~h}$. Further cells were washed with 1X PBS 3 times and counterstained with DAPI (300 $\mathrm{nM})$. Mounting of coverslip was done in Prolong Diamond antifade mounting solution. Images were observed under a 63x oil immersion in Axio observer 7.0 Apotome 2.0 Carl Zeiss microscope.

\section{Confocal- super-resolution microscopy analysis}

To study the actin structures associated with migration, phagocytosis in the presence of ALA, Zeiss LSM 980 with Airy scan 2 in super-resolution mode was used. The immunofluorescence staining for the previously described conditions was carried out with $\beta$-Actin (1:500) and Iba-1 (1:500) proteins to study the microglia activation and actin structures. The super-resolution mode helped to resolve and understand the minute cell structures such as lamellipodia, filopodia, membrane ruffling and polarization state of microglia. The image processing was carried out with Zeiss ZEN 2.3 software.

\section{Western blot}

To understand the protein levels in cells, N9 cells $(3,00$, 000 cells/well) were seeded in 6 well plate. The treatment of extracellular Tau monomer, aggregates along with ALA was given to cells for $24 \mathrm{~h}$. Treatment exposure followed by washing with $1 \mathrm{X}$ PBS. Cell lysis was carried out using radioimmunoprecipitation (RIPA) assay buffer containing protease inhibitors for $20 \mathrm{~min}$ at $4{ }^{\circ} \mathrm{C}$. The cell lysate was centrifuged at $12000 \mathrm{rpm}$ for 20 min. Protein concentration was checked by using Bradford's assay and equal amount of $75 \mu \mathrm{g}$ total proteins for all the treatment groups were loaded on polyacrylamide gel electrophoresis of range $4-20 \%$ and the gel is electrophoretically transferred to polyvinylidene difluoride membrane and kept for primary antibody $\beta$-actin (1: 5000), Iba-1 (1:1000), Arp2 mono (1:1000) and $\beta$-tubulin 
(1:2000) binding for overnight at $4{ }^{\circ} \mathrm{C}$. After the incubation blots washed three times with $1 \mathrm{X}$ PBST $(0.1 \%$ : Tween-20). The secondary antibody was incubated for 1 $h$ at RT. Then the membrane was developed using chemiluminescence detection system. The relative quantification of the protein was carried out with loading control $\beta$-actin $/ \beta$-tubulin in all the treatment group.

\section{Statistical analysis}

All the experiment have been performed in three independent biological replicates. The data was plot using SigmaPlot 10.0 and statistical analysis for microscopic images quantification and western blot experiment quantification was done using One-way ANOVA. The statistical significance between different groups has been performed with Tukey Kramer's post-hoc analysis at 5\% level of significance for multiple group comparison. The test groups were compared with control (untreated) as well as with ALA treated group. The data were considered significant if the mean difference between the groups is greater than Tukey's criteria $\left(\mathrm{X}-\mathrm{X}^{\prime}>\mathrm{T}\right)$, the $p$ value for each group has been calculated and plotted as a star value. The $p$ value indication are done as follows ns- non-significant, " indicates $P \leq 0.05$, ${ }^{* * *}$ indicates $P \leq 0.01$, **** indicates $P \leq 0.001$. The quantification for microscopic images to understand the absolute intensity of desire protein and the corresponding area of microglia was calculated with Zeiss ZEN 2.3 software for image processing. The colocalization analysis between the two proteins from immunofluorescence images (Pearson's coefficient) was done using ImageJ software.

\section{Supplementary Information}

The online version contains supplementary material available at https://doi. org/10.1186/s43556-021-00028-1.

Additional file 1: Figure S1. a-Linolenic acid enhances membrane ruffling in microglia. Figure S2. a-Linolenic acid induces actin polymerization.

\section{Abbreviations}

AD: Alzheimer's disease; ALA: a- Linolenic acid; Arp2/3: Actin related proteins; CNS: Central nervous system; DHA: Docosahexaenoic acid; EPA: Eicosapentaenoic acid; NFTs: Neurofibrillary tangles; PUFAs: Polyunsaturated fatty acids

\section{Acknowledgements}

We are grateful to Chinnathambi lab members for their scientific discussions, helpful suggestions and we highly appreciate critical reading of the manuscript. Author also acknowledges Mrs. Das and Mr. Chidambaram for her/his critical proofreading of the manuscript. Dr. Amarnath and Dr. Rishikanth from Zeiss facility to provide Zeiss LSM 980 with Airyscan 2 for the Confocal Super-Resolution Imaging. SD acknowledges the DBT, Government of India for her fellowship. The authors gratefully acknowledge Professor H. V. Thulasiram, Chemical Biology group at the Division of Organic Chemistry, CSIR-National Chmeical Laboratory for his excellent molecular biology lab and his constant support.

\section{Availability of data materials}

The data is available with the corresponding author and will be provided upon the legitimate request.

\section{Code availability}

Not applicable.

\section{Authors' contributions}

SD and SC performed the experiments and wrote the manuscript. SC conceived, designed, supervised, initial draft, review editing and wrote the paper. All authors read and approved the final paper

\section{Funding}

This project is supported by the in-house CSIR-National Chemical Laboratory grant MLP029526 and MLP101726.

\section{Ethics approval and consent to participate}

Not applicable.

\section{Consent for publication \\ Not applicable.}

\section{Competing interests}

The authors declare no conflicts interests.

Received: 9 August 2020 Accepted: 14 January 2021

Published online: 09 February 2021

\section{References}

1. Swerdlow RH. Pathogenesis of Alzheimer's disease. Clin Interv Aging. 2007; 2(3):347. PMID: 18044185; PMCID: PMC2685260.

2. Ostrowitzki S, Lasser RA, Dorflinger $E$, Scheltens $P$, Barkhof F, Nikolcheva T, et al. A phase III randomized trial of gantenerumab in prodromal Alzheimer's disease. Alzheimers Res Ther. 2017;9(1):95. https://doi.org/10. 1186/s13195-017-0318-y.

3. Köpke E, Tung Y-C, Shaikh S, AdC A, lqbal K, Grundke-lqbal I. Microtubuleassociated protein tau. Abnormal phosphorylation of a non-paired helical filament pool in Alzheimer disease. J Biol Chem. 1993;268(32):24374-84. PMID: 8226987

4. DeVos SL, Corjuc BT, Oakley DH, Nobuhara CK, Bannon RN, Chase A, et al. Synaptic tau seeding precedes tau pathology in human Alzheimer's disease brain. Front Neurosci. 2018;12:267. https://doi.org/10.3389/fnins.2018.00267.

5. Sonawane SK, Chinnathambi S. Prion-like propagation of post-translationally modified tau in Alzheimer's disease: a hypothesis. J Mol Neurosci. 2018; 65(4):480-90. https://doi.org/10.1007/s12031-018-1111-5.

6. Brunello CA, Merezhko M, Uronen R-L, Huttunen HJ. Mechanisms of secretion and spreading of pathological tau protein. Cell Mol Life Sci. 2019; 77(9):1-24. https://doi.org/10.1007/s00018-019-03349-1.

7. Sebastián-Serrano Á, Diego-García D, Díaz-Hernández M. The neurotoxic role of extracellular tau protein. Int J Mol Sci. 2018;19(4):998. https://doi.org/10. 3390/ijms19040998.

8. Majerova P, Zilkova M, Kazmerova Z, Kovac A, Paholikova K, Kovacech B, et al. Microglia display modest phagocytic capacity for extracellular tau oligomers. J Neuroinflammation. 2014;11(1):161. https://doi.org/10.1186/ s12974-014-0161-z.

9. Bolós M, Llorens-Martín M, Jurado-Arjona J, Hernández F, Rábano A, Avila J. Direct evidence of internalization of tau by microglia in vitro and in vivo. J Alzheimers Dis. 2016;50(1):77-87. https://doi.org/10.3233/JAD-150704.

10. Hopp SC, Lin Y, Oakley D, Roe AD, DeVos SL, Hanlon D, et al. The role of microglia in processing and spreading of bioactive tau seeds in Alzheimer's disease. J Neuroinflammation. 2018;15(1):1-15. https://doi.org/10.1186/ s12974-018-1309-z.

11. Perea JR, Llorens-Martín M, Ávila J, Bolós M. The role of microglia in the spread of tau: relevance for tauopathies. Front Cell Neurosci. 2018;12:172. https://doi.org/10.3389/fncel.2018.00172.

12. Das R, Chinnathambi S. Microglial priming of antigen presentation and adaptive stimulation in Alzheimer's disease. Cell Mol Life Sci. 2019;76(19): 1 14. https://doi.org/10.1007/s00018-019-03132-2.

13. Lively $\mathrm{S}$, Schlichter LC. The microglial activation state regulates migration and roles of matrix-dissolving enzymes for invasion. J Neuroinflammation. 2013;10(1):843. https://doi.org/10.1186/1742-2094-10-75. 
14. Das R, Chinnathambi S. Actin-mediated microglial Chemotaxis via G-protein coupled Purinergic receptor in Alzheimer's disease. Neuroscience. 2020;448: 325-36. https://doi.org/10.1016/j.neuroscience.2020.09.024.

15. Schachtner $\mathrm{H}$, Calaminus SD, Thomas SG, Machesky LM. Podosomes in adhesion, migration, mechanosensing and matrix remodeling. Cytoskeleton. 2013;70(10):572-89. https://doi.org/10.1002/cm.21119.

16. Lim HM, Woon H, Han JW, Baba Y, Kurosaki T, Lee MG, et al. UDP-induced phagocytosis and ATP-stimulated chemotactic migration are impaired in STIM1-/- microglia in vitro and in vivo. Mediat Inflamm. 2017;8158514. https://doi.org/10.1155/2017/8158514.

17. Freeman SA, Grinstein S. Phagocytosis: receptors, signal integration, and the cytoskeleton. Immunol Rev. 2014;262(1):193-215. https://doi.org/10.1111/ imr.12212.

18. Uhlemann R, Gertz K, Boehmerle W, Schwarz T, Nolte C, Freyer D, et al. Actin dynamics shape microglia effector functions. Brain Struct Funct. 2016; 221(5):2717-34. https://doi.org/10.1007/s00429-015-1067-y.

19. Svitkina T. The actin cytoskeleton and actin-based motility. Cold Spring Harb Perspect Biol. 2018;10(1):a018267. https://doi.org/10.1101/cshperspect. a018267.

20. Ohsawa K, Imai Y, Kanazawa H, Sasaki Y, Kohsaka S. Involvement of Iba1 in membrane ruffling and phagocytosis of macrophages/microglia. J Cell Sci. 2000;113(17):3073-84. PMID: 10934045.

21. Franco-Bocanegra DK, McAuley C, Nicoll JA, Boche D. Molecular mechanisms of microglial motility: changes in ageing and Alzheimer's disease. Cells. 2019;8(6):639. https://doi.org/10.3390/cells8060639.

22. Mandrekar-Colucci S, Landreth GE. Microglia and inflammation in Alzheimer's disease. CNS Neurol Disorder Drug Target. 2010;9(2):156-67. https://doi.org/10.2174/187152710791012071.

23. Lee HN, Sim KM, Kim H, Ju J, Pae AN, Park J-B, et al. A $\beta$ modulates actin cytoskeleton via SHIP2-mediated phosphoinositide metabolism. Sci Rep. 2019;9(1):1-12. https://doi.org/10.1038/s41598-019-51914-2

24. Das R, Balmik AA, Chinnathambi S. Phagocytosis of full-length tau oligomers by actin-remodeling of activated microglia. J Neuroinflammation. 2020;17(1): 1-15. https://doi.org/10.1186/s12974-019-1694-y.

25. Fang G, Shi B, Wu K, Chen S, Gao X, Xiao S, et al. The protective role of endogenous $\mathrm{n}-3$ polyunsaturated fatty acids in tau Alzheimer's disease mouse model. Int J Neurosci. 2019;129(4):325-36. https://doi.org/10.1080/ 00207454.2018 .1533824 .

26. Laye S, Nadjar A, Joffre C, Bazinet RP. Anti-inflammatory effects of omega-3 fatty acids in the brain: physiological mechanisms and relevance to pharmacology. Pharmacol Rev. 2018;70(1):12-38. https://doi.org/10.1124/pr.117.014092

27. Desale SE, Chinnathambi S. Role of dietary fatty acids in microglial polarization in Alzheimer's disease. J Neuroinflammation. 2020;17(1):1-14 https://doi.org/10.1186/s12974-020-01742-3.

28. Schmidt S, Willers J, Riecker S, Möller K, Schuchardt JP, Hahn A. Effect of omega-3 polyunsaturated fatty acids on the cytoskeleton: an open-label intervention study. Lipids Health Dis. 2015;14(1):4. https://doi.org/10.1186/1476-511X-14-4.

29. Hjorth E, Zhu M, Toro VC, Vedin I, Palmblad J, Cederholm T, et al. Omega-3 fatty acids enhance phagocytosis of alzheimer's disease-related amyloid- $\beta$ 42 by human microglia and decrease inflammatory markers. J Alzheimers Dis. 2013;35(4):697-713. https://doi.org/10.3233/JAD-130131.

30. Desale SE, Chinnathambi S. a-Linoleanic acid modulates phagocytosis of extracellular Tau and induces microglial migration by actin-remodeling. bioRxiv. 2020

31. Fuentes NR, Kim E, Fan Y-Y, Chapkin RS. Omega-3 fatty acids, membrane remodeling and cancer prevention. Mol Asp Med. 2018;64:79-91. https:// doi.org/10.1016/..mam.2018.04.001.

32. Masters TA, Pontes B, Viasnoff V, Li Y, Gauthier NC. Plasma membrane tension orchestrates membrane trafficking, cytoskeletal remodeling, and biochemical signaling during phagocytosis. Proc Natl Acad Sci. 2013;110(29): 11875-80. https://doi.org/10.1073/pnas.1301766110.

33. Lowry JR, Marshall N, Wenzel TJ, Murray TE, Klegeris A. The dietary fatty acids a-Linolenic acid (ALA) and linoleic acid (LA) selectively inhibit microglial nitric oxide production. Mol Cell Neurosci. 2020; 109: 103569. https://doi.org/10.1016/j.mcn.2020.103569.

34. Janda E, Boi L, Carta AR. Microglial phagocytosis and its regulation: a therapeutic target in Parkinson's disease? Front Mol Neurosci. 2018;11:144. https://doi.org/10.3389/fnmol.2018.00144.

35. Desale SE, Dubey T. Chinnathambi S. a-Linolenic acid inhibits Tau aggregation and modulates Tau conformation. Int J Biol Macromol. 2020; 166: 687-93. https://doi.org/10.1016/j.ijbiomac.2020.10.226.
36. Rosales C, Uribe-Querol E. Phagocytosis: a fundamental process in immunity. Biomed Res Int. 2017;9042851. https://doi.org/10.1155/2017/ 9042851.

37. Krause M, Gautreau A. Steering cell migration: lamellipodium dynamics and the regulation of directional persistence. Nat Rev Mol Cell Biol. 2014;15(9): 577-90. https://doi.org/10.1038/nrm3861.

38. Hovens IB, Nyakas C, Schoemaker RG. A novel method for evaluating microglial activation using ionized calcium-binding adaptor protein-1 staining: cell body to cell size ratio. Neuroimmunol Neuroinflammation. 2014;1 (2):82-8. https://doi.org/10.1038/nrm3861.

39. Doughman RL, Firestone AJ, Wojtasiak ML, Bunce MW, Anderson RA. Membrane ruffling requires coordination between type la phosphatidylinositol phosphate kinase and Rac signaling. J Biol Chem. 2003; 278(25):23036-45. https://doi.org/10.1074/jbc.M211397200.

40. Huang C-C, Wang S-Y, Lin L-L, Wang P-W, Chen T-Y, Hsu W-M, et al. Glycolytic inhibitor 2-deoxyglucose simultaneously targets cancer and endothelial cells to suppress neuroblastoma growth in mice. Dis Model Mech. 2015;8(10):1247-54. https://doi.org/10.1242/dmm.021667.

41. Taylor KL, Taylor RJ, Richters KE, Huynh B, Carrington J, McDermott ME, et al. Opposing functions of F-BAR proteins in neuronal membrane protrusion, tubule formation, and neurite outgrowth. Life Sci Alliance. 2019;2(3): e201800288. https://doi.org/10.26508/Isa.201800288.

42. Chhabra ES, Higgs HN. The many faces of actin: matching assembly factors with cellular structures. Nat Cell Biol. 2007;9(10):1110-21. https://doi.org/10. 1038/ncb1007-1110

43. Bailly M, Macaluso F, Cammer M, Chan A, Segall JE, Condeelis JS. Relationship between Arp2/3 complex and the barbed ends of actin filaments at the leading edge of carcinoma cells after epidermal growth factor stimulation. J Cell Biol. 1999;145(2):331-45. https://doi.org/10.1083/jcb. 145.2.331.

44. Spires-Jones TL, Hyman BT. The intersection of amyloid beta and tau at synapses in Alzheimer's disease. Neuron. 2014;82(4):756-71. https://doi.org/ 10.1016/j.neuron.2014.05.004

45. Lee CD, Landreth GE. The role of microglia in amyloid clearance from the AD brain. J Neural Transm. 2010;117(8):949-60. https://doi.org/10.1007/ s00702-010-0433-4.

46. Sheffield LG, Marquis JG, Berman NE. Regional distribution of cortical microglia parallels that of neurofibrillary tangles in Alzheimer's disease. Neurosci Lett. 2000;285(3):165-8. https://doi.org/10.1016/s03043940(00)01037-5.

47. Sasaki A, Kawarabayashi T, Murakami T, Matsubara E, Ikeda M, Hagiwara H, et al. Microglial activation in brain lesions with tau deposits: comparison of human tauopathies and tau transgenic mice TgTauP301L. Brain Res. 2008; 1214:159-68. https://doi.org/10.1016/j.brainres.2008.02.084.

48. Španić E, Langer Horvat L, Hof PR, Simic G. Role of microglial cells in Alzheimer's disease tau propagation. Front Aging Neurosci. 2019;11:271. https://doi.org/10.3389/fnagi.2019.00271.

49. Kawano A, Ariyoshi W, Yoshioka Y, Hikiji H, Nishihara T, Okinaga T. Docosahexaenoic acid enhances M2 macrophage polarization via the p38 signaling pathway and autophagy. J Cell Biochem. 2019;120(8):12604-17. https://doi.org/10.1002/jcb.28527.

50. Svitkina TM. Ultrastructure of protrusive actin filament arrays. Curr Opin Cell Biol. 2013;25(5):574-81. https://doi.org/10.1016/j.ceb.2013.04.003.

51. May RC, Machesky LM. Phagocytosis and the actin cytoskeleton. J Cell Sci. 2001;114(6):1061-77.

52. Balmik AA, Chidambaram H, Dangi A, Marelli UK, Chinnathambi S. HDAC6 ZnF UBP as the modifier of tau structure and function. Biochemistry. 2020; 59(48):4546-62. https://doi.org/10.1021/acs.biochem.0c00585.

53. Sonawane SK, Balmik AA, Boral D, Ramasamy S, Chinnathambi S. Baicalein suppresses repeat tau fibrillization by sequestering oligomers. Arch Biochem Biophys. 2019;675:108119. https://doi.org/10.1016/j.abb.2019.108119.

54. Wilson DM, Binder LI. Free fatty acids stimulate the polymerization of tau and amyloid beta peptides. In vitro evidence for a common effector of pathogenesis in Alzheimer's disease. Am J Pathol. 1997;150(6):2181. PMID: 9176408; PMCID: PMC1858305.

\section{Publisher's Note}

Springer Nature remains neutral with regard to jurisdictional claims in published maps and institutional affiliations. 